\title{
A ZEUTHEN SEGRE FORMULA FOR EVEN DIMENSIONAL SUBMANIFOLDS OF REAL PROJECTIVE SPACE
}

\author{
by J. W. BRUCE
}

\section{(Received 19 September, 1981)}

In this paper we generalise results of Craveiro de Carvalho ([3]) in two ways. First we prove the following fact.

Proposition 1. Given any smooth submanifold $M$ of real projective space $\mathbb{P}^{n}$, for $L$ in an open dense subset of the space of codimension 2 subspaces of $\mathbb{P}^{n}$ we have

(a) L meets $M$ transversally and

(b) the pencil of hyperplanes through $L$ have at worst Morse $\left(A_{1}\right)$ contact with $M$.

Now suppose that the dimension of $M$ is even, $p \in M$ and a hyperplane $H$ in the pencil is tangent to $M$ at $p$. We can measure the contact between $M$ and $H$ at $p$ by considering the height function in some local coordinate system in a direction normal to $H$ on $M$ at $p$. This contact is defined up to contact equivalence (see [2]) and if it is of type $A_{1}$ this means we only have a Morse local normal form up to sign. However since $M$ is of even dimension the sign of the determinant of the Hessian is well defined and we denote it by $\varepsilon_{p}$. (In the case when $M$ is a hypersurface $\varepsilon_{p}$ is the sign of the Gauss curvature of $M$ at $p$ in any affine chart).

Proposition 2. If $\operatorname{dim} M$ is even and $L$ determines a generic pencil then $\chi(M)=$ $\chi(M \cap L)+\sum_{p \in M} \varepsilon_{p}$, where $\chi(X)$ denotes the Euler characteristic of $X$.

Proof of Proposition 1. We first prove part (a). Since openness follows from general principles it is enough to prove density. If $\mathbb{P}^{n}$ denotes the dual space of hyperplanes in $\mathbb{P}^{n}$ we claim that it is enough to prove that an (open) dense subset of $\mathscr{P}^{n}$ meets any submanifold $M \subset \mathbb{P}^{n}$ transversally. For if $L$ is given by $x_{0}=x_{1}=0$ we may choose a hyperplane arbitrarily near $x_{0}=0$, say $H_{0}$, which is transverse to $M$ and a hyperplane arbitrarily near $x_{1}=0$, say $H$, which is transverse to $M \cap H_{0}$. Then $H_{0} \cap H_{1}$ meets $M$ transversally and can be made arbitrarily near $L$.

But consider $\Gamma(M)=\left\{(x, H) \in M \times \check{\mathbb{P}}^{n}: x \in H\right\}$; clearly $\Gamma(M)$ is a smooth manifold and if $\pi: \Gamma(M) \rightarrow \check{P}^{n}$ is the natural projection then $H$ is a regular value of $\pi$ if and only if $H$ is transverse to $M$. By Sard's theorem the set of critical values of $\pi$ is of measure zero in $\mathscr{P}^{n}$ (and by the same general principles as above it is a closed set) whence the result.

Now suppose that $L=\left\{x_{0}=x_{1}=0\right\}$ meets $M$ transversally as does $H_{0}=\left\{x_{0}=0\right\}$. We consider the family of functions on $M-H_{0}, F:\left(M-H_{0}\right) \times \mathbb{R}^{n+1} \rightarrow \mathbb{R}$ given by

$$
F_{\mathbf{a}}(x)=F(x, \mathbf{a})=\frac{x_{i}+\sum_{0}^{n} a_{i} x_{i}}{x_{0}}
$$

Glasgow Math. J. 24 (1983) 97-99. 
(which is well defined). Fixing a this function measures the contact of the pencil of hyperplanes determined by $x_{0}=x_{1}+\sum_{0}^{n} a_{i} x_{i}=0$ with $M$. We want to show that, for most values of $\mathbf{a}$, the function $F_{\mathrm{a}}$ has only Morse singularities. To do this we need to consider the 2 -jets of the $F_{\mathbf{a}}$, and ideally we would prove that the natural jet extension

$$
j_{1}^{2} F:\left(M-H_{0}\right) \times \mathbb{R}^{n+1} \rightarrow J^{2}\left(M-H_{0}, \mathbb{R}\right)
$$

is transverse to the natural stratification of the 2 -jets by their rank. Then, by a lemma of Thom, for almost all a (i.e. all a outside a set of measure zero) $j^{2} F_{\mathbf{a}}$ will be transverse to this stratification and hence $F_{\mathrm{a}}$ will only have Morse singularities. Unfortunately we cannot use this version of Thom's lemma, as we shall see but a sharpened version due to Mather does prove the result.

To compute the image of the differential of $j_{1}^{2} F$ at $(y, \mathbf{b})$ it is clear that we may as well take $\mathbf{b}=\mathbf{0}$ and $y=(1: 0, \ldots ; 0)$ with $M$ given near $y$ by $x_{0}=1, x_{1}=$ $f_{1}\left(x_{k+1}, \ldots, x_{n}\right), \ldots, x_{k}=f_{k}\left(x_{k+1}, \ldots, x_{n}\right)$, where $M$ is of dimension $n-k$.

Working in the fibre of the jet space (with no constants) $J_{0}^{2}(n-k, 1)$ we obtain the following vectors.

(a) From $\partial / \partial a_{0}$ we get 1 (which is disregarded of course).

(b) From $\partial / \partial a_{j}$, where $1 \leqslant j \leqslant k$, we get $f_{j}$.

(c) From $\partial / \partial a_{j}$, where $k+1 \leqslant j \leqslant n$, we get $x_{j}$.

The tangent space to the orbit of $j^{2} f_{1}=j_{1}^{2} F_{0}$ is spanned by $\mu J\left(f_{1}\right)$ where $\mu$ is the maximal ideal $\left\langle x_{k+1}, \ldots, x_{n}\right\rangle$ and $J\left(f_{1}\right)$ is the Jacobian ideal spanned by the partial derivatives of $f_{1}$.

From the $M$ space and this tangent space to the orbit we obtain simply $\boldsymbol{J}\left(f_{1}\right)$.

Now the theorem of Mather $\left(\left[4\right.\right.$, p. 230]) asserts that if either (a) the jet extension $j_{1}^{2} F$ is transverse to the orbits in $J_{0}^{2}(n-1,1)$ or (b) $j_{1}^{2} F$ is more transverse than $j_{1}^{2} F_{b}$, then for almost all a the map $j_{1}^{2} F_{\mathrm{a}}$ is transverse to these orbits, which is the result we require. Thus we want to show that $J\left(f_{1}\right) / \mathcal{M}^{3}=\left(J\left(f_{1}\right)+\operatorname{Sp}\left\{x_{k+1}, \ldots, x_{n}, f_{j}\right\}\right) / \mathcal{M}^{3}$ implies $j_{1}^{2} F$ is transverse to the orbits. But if $J\left(f_{1}\right) \supset \operatorname{Sp}\left\{x_{k+1}, \ldots, x_{n}\right\}\left(\bmod . \mathcal{M}^{3}\right)$, then $f_{1}$ must be Morse and transversality of $j_{1}^{2} F$ (indeed $j_{1}^{2} F_{0}$ ) is obvious. Thus for a arbitrarily close to $0 \in \mathbb{R}^{n+1}$ we have $F_{\mathrm{a}}: M-H_{0} \rightarrow \mathbb{B}$ a Morse function, and the pencil determined by $x_{0}=x_{1}+\sum a_{i} x_{i}=0$ is of the required type. The proof is complete.

Remark. It is not difficult to show we can also arrange for no planes in the pencil to be bitangent to $M$, i.e. the critical values of $F_{\mathrm{a}}$ to be distinct.

Proof of Proposition 2. Let us denote the axis of our generic pencil by $L$, which we may suppose is given by $x_{0}=x_{1}=0$. Following [1] we blow $\mathbb{P}^{n}$ up along the axis $L$, i.e. we consider $\Delta=\left\{(x, t) \in \mathbb{P}^{n} \times \mathbb{P}^{1}: x_{0} t_{1}+x_{1} t_{0}=0\right\}$, and if $\pi_{1}: \Delta \rightarrow \mathbb{P}^{n}$ is the natural projection we set $\tilde{M}=\pi_{1}^{-1}(M) \subset \Delta$. Since $L$ meets $M$ transversally, $\tilde{M}$ is smooth and $\pi_{1}: \bar{M}-\pi_{1}^{-1}(L) \rightarrow$ $M-L$ is a diffeomorphism. Becaușe $L$ determines a generic pencil projection to the second factor, $\pi_{2}: \tilde{M} \rightarrow \mathbb{P}^{1}$ has only Morse singularities. If $t$ is a regular value of $\pi_{2}$ and $U$ 
an open neighbourhood of $t$ whose closure $\bar{U}$ consists of regular values, then $\pi_{2}: \tilde{M}-$ $\pi_{2}^{-1}(U) \rightarrow \mathbb{P}^{1}-U$ is Morse and $\chi\left(\tilde{M}-\pi_{2}^{-1}(U)\right)=\chi\left(\pi_{2}^{-1}(t)\right)+\sum_{p \in M} \varepsilon_{p}$, using standard results from elementary Morse theory. Since $\pi_{2}^{-1}(\bar{U}) \cong \bar{U} \times \pi_{2}^{-1}(t)$ from the Mayer-Vietoris sequence one easily finds that $\chi(\tilde{M})=\sum_{p \in M} \varepsilon_{p}$.

Now $\pi_{1}$ is a diffeomorphism away from $\pi_{1}^{-1}(L)$, and over points of $M \cap L$ it has $\mathbb{P}^{1}$ as fibres; $M \cap L$ is of codimension 2 in $M$ so if $T$ denotes a tubular neighbourhood of $M \cap L$ in $M$ we have $\chi(M)=\chi(M-L)+\chi(M \cap L)-\chi(\partial T)$. But, as $\partial T$ is an $S^{1}$ bundle, $\chi(\partial T)=0$ and $\chi(M)=\chi(M-L)+\chi(M \cap L)$. Similarly

$$
\chi(\tilde{M})=\sum_{p \in M} \varepsilon_{p}=\chi\left(\tilde{M}-\pi_{1}^{-1}(M \cap L)+\chi\left(\pi_{1}^{-1}(M \cap L)\right)-\chi(\partial \tilde{T}),\right.
$$

where $\tilde{T}$ is a tubular neighbourhood of $\chi_{1}^{-1}(M \cap L)$ in $M$. But $\partial \tilde{T} \rightarrow \pi_{1}^{-1}(M \cap L)$ is a 2-fold cover, $\pi_{1}^{-1}(M \cap L) \rightarrow M \cap L$ is an $S^{1}$ bundle and $\tilde{M}-\pi_{1}^{-1}(M \cap L) \rightarrow M-L$ is a diffeomorphism, so

$$
\sum_{p \in M} \varepsilon_{p}=\chi(M-L)=\chi(M)-\chi(M \cap L)
$$

whence the result.

\section{REFERENCES}

1. A. Androtti and T. Frankel, The Lefschetz hyperplane theorems in Global Analysis-Papers in Honour of K. Kodaira. (1969).

2. J. W. Bruce, P. J. Giblin and C. G. Gibson, Caustics by reflection. Topology. To appear.

3. F. J. Craveiro de Carvalho, Immersed surfaces and pencils of planes in 3-space. Glasgow Math. J. 22 (1981), 133-136.

4. J. Mather, Generic projections. Annals of Maths., 98 (1973), 226-245.

5. J. Milnor, Morse theory, Annals of Maths. Studies 51. (Princeton University Press, 1963).

UNIVERSTTY COLLEGE,

CORK, EIRE 